\title{
How evidence-based is an 'evidence-based parenting program'? A PRISMA systematic review and meta-analysis of Triple $P$
}

Philip Wilson ${ }^{1 *}$, Robert Rush ${ }^{2}$, Susan Hussey ${ }^{3}$, Christine Puckering ${ }^{4}$, Fiona Sim ${ }^{4}$, Clare S Allely ${ }^{4}$, Paul Doku Alex McConnachie ${ }^{5}$ and Christopher Gillberg ${ }^{4}$

\begin{abstract}
Background: Interventions to promote positive parenting are often reported to offer good outcomes for children but they can consume substantial resources and they require rigorous appraisal.

Methods: Evaluations of the Triple P parenting program were subjected to systematic review and meta-analysis with analysis of biases. PsychInfo, Embase and Ovid Medline were used as data sources. We selected published articles reporting any child-based outcome in which any variant of Triple P was evaluated in relation to a comparison condition. Unpublished data, papers in languages other than English and some book chapters were not examined. Studies reporting Eyberg Child Behavior Inventory or Child Behavior Checklist scores as outcomes were used in the meta-analysis.

Results: A total of 33 eligible studies was identified, most involving media-recruited families. Thirty-one of these 33 studies compared Triple P interventions with waiting list or no-treatment comparison groups. Most papers only reported maternal assessments of child behavior. Twenty-three papers were incorporated in the meta-analysis. No studies involved children younger than two-years old and comparisons of intervention and control groups beyond the duration of the intervention were only possible in five studies. For maternally-reported outcomes the summary effect size was 0.61 (95\% 0.42 , 0.79). Paternally-reported outcomes following Triple $\mathrm{P}$ intervention were smaller and did not differ significantly from the control condition (effect size $0.42(95 \% \mathrm{Cl}-0.02,0.87)$ ). The two studies involving an active control group showed no between-group differences. There was limited evidence of publication bias, but there was substantial selective reporting bias, and preferential reporting of positive results in article abstracts. Thirty-two of the 33 eligible studies were authored by Triple-P affiliated personnel. No trials were registered and only two papers contained conflict of interest statements.
\end{abstract}

Conclusions: In volunteer populations over the short term, mothers generally report that Triple P group interventions are better than no intervention, but there is concern about these results given the high risk of bias, poor reporting and potential conflicts of interest. We found no convincing evidence that Triple $P$ interventions work across the whole population or that any benefits are long-term. Given the substantial cost implications, commissioners should apply to parenting programs the standards used in assessing pharmaceutical interventions. See related commentary: http://www.biomedcentral.com/1741-7015/10/145

Keywords: parenting, public health, child psychology, behavioral family intervention, systematic review, metaanalysis

\footnotetext{
* Correspondence: p.wilson@abdn.ac.uk

${ }^{1}$ Centre for Rural Health, University of Aberdeen, Centre for Health Sciences,

Old Perth Rd, Inverness IV2 3JH, Scotland

Full list of author information is available at the end of the article
} 


\section{Introduction \\ Rationale}

Problems in effective parenting are increasingly seen as a significant public health issue [1] and public policy has come to reflect this. The Positive Parenting Programme (Triple P) [2] is a multi-level behavioral family intervention which has been proposed $[3,4]$ and used $[5,6]$ in recent years on a whole-population basis as a public health intervention in addition to its use on a more targeted basis. Many administrative entities (cities or counties) throughout the world have adopted or are in the process of adopting the program on a large scale, with substantial cost implications [7]. UK National Institute for Clinical Excellence guidelines suggest Triple P is an effective educational intervention for parents of children with conduct disorder, a recommendation which carries considerable weight in policy and purchasing decisions in England [8].

The evidence base for Triple $\mathrm{P}$ appears to be extensive, with more than 200 publications and a large number of published randomized trials. There are four existing meta-analyses of the program [9-12], uniformly reporting positive effects on child behavior, but these reviews did not make systematic attempts to analyze risk of bias beyond the differing effect sizes attributable to different informants $[9,11]$. Moderators of effectiveness, such as severity of presenting problems, intensity of intervention and age/gender of the child, were assessed in three reviews $[9,11,12]$. There is some doubt about the effectiveness of Triple $\mathrm{P}$ in deprived communities [11], with lone parents [13] and among younger children, and the overall impact at population level has not been examined in detail. Much of the published work is authored by affiliates of the Triple P organization putting the independence of the evidence in a less favorable position.

We have used Preferred Reporting Items for Systematic Reviews and Meta-Analyses (PRISMA) guidelines [14] to examine reporting and other biases in a systematic way and to delineate any gaps in the evidence base supporting Triple P. We have focused on child-based outcomes in this review since the ultimate aim of parenting programs is to improve children's wellbeing.

\section{Objectives}

We examined the published data to:

-Identify characteristics of the populations in which Triple $\mathrm{P}$ interventions have been subject to investigation

- Clarify which comparison conditions were used in Triple P evaluations

- Identify child-based outcome measures and which informants provided outcome data
- Examine critically the design of studies in which comparisons with alternative interventions have been reported

- Clarify any contribution of publication bias to the existing meta-analyses through examination of trial registry entries, funnel plots, and meta-regression approaches

- Clarify any contribution of outcome reporting bias and selective reporting of results in article abstracts

\section{Methods}

\section{Protocol and registration}

We did not register the protocol for this review.

\section{Eligibility criteria}

Published articles in which any level of Triple P (or a precursor behavioral family intervention from the same group of authors) was used, in which any (non-Triple-P) comparison condition was employed, and in which a quantitative child-based outcome was reported, were eligible for inclusion in the systematic review. Criteria for the meta-analysis were more restrictive: eligible studies were randomized controlled trials (RCTs) reporting Child Behavior Checklist or Eyberg Child Behavior Inventory scores for intervention and comparison groups.

Journal articles published in English before September 2011 were eligible for inclusion. We also examined book chapters, whole books and electronic documents available locally and through the United Kingdom's interlibrary loan system.

\section{Information sources}

We searched databases PsycINFO 1970- August 2011, Embase 1980- August week 32011 and Ovid Medline 1950-August week 3 2011. We also included all journal articles, books and book chapters listed on the Parenting and Family Support Centre Triple-P database at the University of Queensland [15] (accessed 16 September 2009 and 29 August 2011) and relevant secondary references from the four available systematic reviews $[9,11,12,16]$.

\section{Search}

A search was carried out on 29 August 2011 using the following strategy:

Keywords = "bfi" or "Behav\$ Family Intervention" or ["parenting" and "Triple"] or ["positive" and "parenting"].

\section{Study selection}

The study selection process is illustrated in Figure 1. 


\section{Identification}

No of records identified through database searching

4477

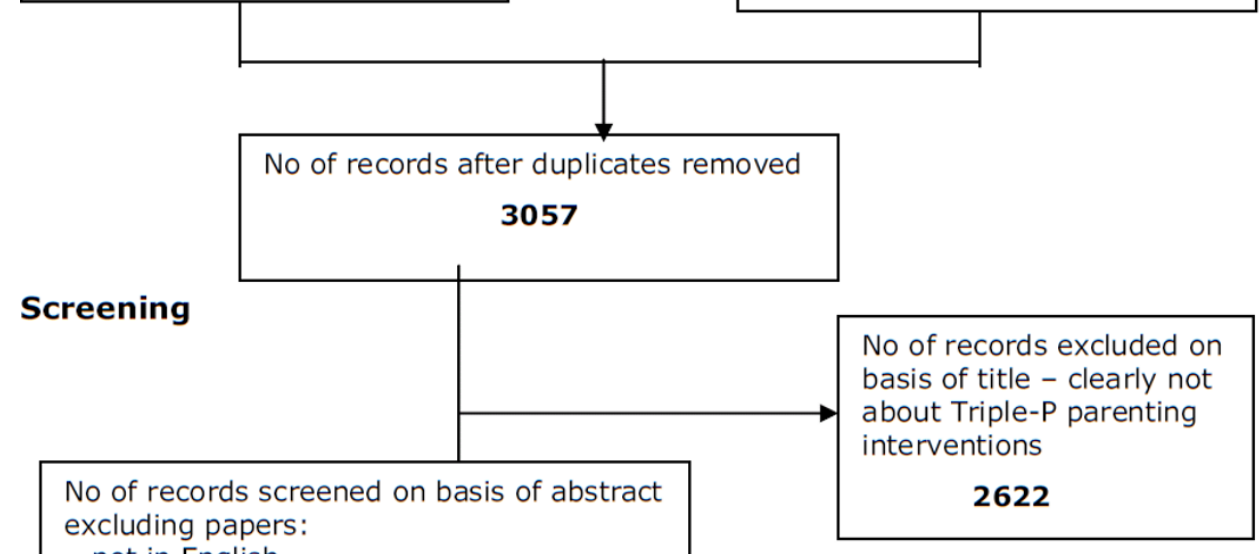

excluding papers:

- not in English

- review paper only

- clearly not Triple P intervention studies

- clearly without a comparison

group

- clearly without a quantifiable child

outcome

435

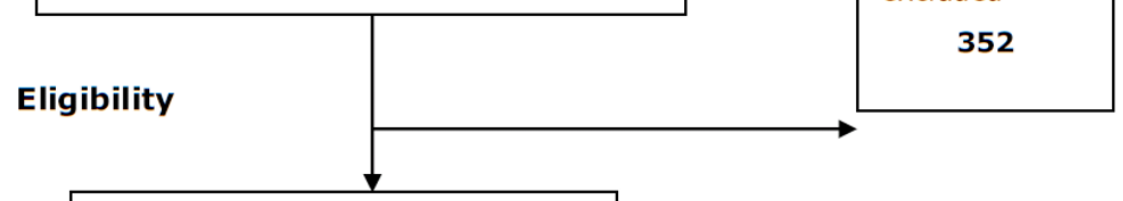

No of full text articles assessed for eligibility
83

Included
No of studies included in qualitative synthesis

\section{3}

No of studies included in quantitative synthesis (meta-analysis)

\section{3}

No of additional records identified through other sources

228
No of full text articles excluded, with reasons (may be more than one reason for exclusion per article)

\section{9}

- not an intervention study 23

-not about Triple $\mathrm{P}$ or a related intervention 22

- no comparison group 34

- no quantifiable child outcome 20

- no original data 11 
Screening:

In the first stage, papers which were clearly not:

- intervention studies or

- studies about the Triple-P parenting program or one of its precursors were excluded on the basis of title alone.

For the next stage papers were rejected which:

- were not published in the English language

- were not intervention studies

- were not conducted using a comparison group

- did not report a quantifiable child outcome.

In addition, review papers and book chapters which were clearly reviews were excluded.

Full documents were obtained for the remaining records.

Papers were rejected at this stage if they:

- were not intervention studies

- were not conducted using a non-Triple P comparison group

- did not report a quantifiable child outcome

- did not use Triple P or one of its precursors as an intervention

- did not report original data.

Eligible papers were tabulated and used in the qualitative synthesis.

For the meta-analysis, papers from randomized trials reporting the two most commonly used outcome measures, the Intensity scale of the Eyberg Child Behavior Inventory [17] (ECBI-I) and the Externalizing Behavior subscale of the Child Behavior Checklist (CBCL) [18] were used. These are the outcome measures reported in other meta-analyses of Triple-P child-based outcomes and are applicable to children 2- to 16-years old. Other child-based outcomes (apart from the Problem subscale of the ECBI and the Internalizing subscale of the CBCL) were reported in too few studies to allow meaningful meta-analysis. Reductions in ECBI-I and CBCL scores represent improvement. Scores on the ECBI and CBCL subscales not reported here generally mirrored those that we have reported but effect sizes were usually of lesser magnitude.

\section{Data collection process}

Data were collected, with permission, onto a form based on that used by the Scottish Intercollegiate Guideline Network [19] (accessed 11 October 2012). For each paper, two of the authors completed the data collection form. If authors disagreed, a third author adjudicated.
As our analysis concerned only published data, we did not seek to obtain further data from investigators.

\section{Data items}

The following variables were assessed:

- Numbers of patients or families included in the study

- Main characteristics of the patient population

- Nature of the intervention being investigated

- Which outcomes were compared across groups

- Nature of the control or comparison group

- Length of follow-up

- Nature of child-based outcome measure(s) used in the study

- Which outcomes were reported in article Results and Abstract sections

- Whether a principal outcome measure was prespecified

- Whether a power calculation was included

- Whether the assignment of subjects to treatment groups was randomized

- Whether an adequate concealment method was used (RCTs only)

- Whether reporters of the child-based outcomes were blind to treatment allocation

- Whether treatment and control groups were similar at baseline

- Dropout rates for participants recruited into each arm of the study

- Whether group differences were analyzed by intention to treat

- Whether subgroup analyses were performed

- Mean and standard deviation of post-intervention child-based outcome measures (for meta-analysis)

- Whether a statement of study funding was included

- Affiliations of authors

- Whether a conflict of interest statement was included

- Whether trials were registered with a public trials registry.

\section{Risk of bias in individual studies}

Outcome reporting bias within eligible studies was reported qualitatively. Numerical summaries were made of the likelihood of statistically significant and non-significant results being equally reported in the Results and Abstract sections of published papers.

\section{Summary measures}

The effect size (ES) for each study included in the metaanalysis was estimated using the standardized mean 
difference (SMD), with post-intervention mean and pooled standard deviation. Hedges g, under a random effects modelling approach, was used to obtain unbiased estimates of ESs. From studies with more than one treatment group, or subgroups reported separately, an averaged effect was derived based on sample size, standard deviation and mean.

\section{Synthesis of results}

Both fixed and random effects models were generated, but the resulting models were very similar and only the random effects model is reported here. Random effects models assume that treatment effects may differ between studies, and this assumption has face validity given that treatment intensities and types of participants varied between studies.

Variation in SMDs attributable to heterogeneity was assessed with the I-squared statistic (that is, the percentage of between study heterogeneity attributable to variability in the true treatment effect, rather than sampling variation).

\section{Risk of bias across studies}

Publication bias was assessed with funnel plots which illustrate the possibility of selective publication of small studies with positive results. Egger's regression based adjustment method was used on the data presented on funnel plots.

\section{Additional analyses}

We planned sensitivity analyses in relation to authorship (Triple-P affiliated versus non-Triple-P affiliated). We also planned a subgroup analysis of data obtained on child behavior from sources other than the mother or principal carer (for example, fathers, teachers, independent observers).

In order to assess whether baseline symptom severity moderated treatment effects, we undertook a random effects meta-regression to investigate the association of baseline (pre-intervention) values with ES, examining only those studies which employed the most commonly used outcome measure - the ECBI Intensity scale score.

\section{Results}

\section{Study selection}

The selection process is illustrated in Figure 1.

\section{Study characteristics}

The main characteristics of the studies are presented in additional file 1 . Most of the studies (26/33) used a waiting list control condition where treatment was offered immediately after the post-intervention assessment. This design precluded control group follow-up beyond the end of the intervention. Comparisons of intervention and control groups beyond the duration of the intervention were only possible in five studies.

\section{Risk of bias within studies}

Data on the risk of bias of each study are presented in Table 1.

No studies were registered with national or international trials registries. All the studies apart from two $[6,20]$ used individual or cluster $[5,13,21,22]$ random assignment to the study group, but the mechanism of randomization was only reported in the minority of studies. No papers reported a pre-specified principal outcome measure, and no power calculations based on specific outcome measures were reported. Four papers reported a power calculation based on a general ES [23-26]. All eligible papers appeared to be co-authored by a Triple-P affiliated author, apart from one [26], although it was difficult to establish affiliation in some cases. We were, therefore, not able to conduct sensitivity analyses in relation to authorship. Conflict of interest statements were found in two papers: one [13], where 'no conflict' was reported and another [27] where royalty payments to authors were mentioned.

There is substantial risk of outcome reporting bias. Between papers, there is inconsistent reporting of subscale results within the Strengths and Difficulties Questionnaire, the ECBI, the Family Observation Schedule and the Developmental Behavior Checklist. In some papers all subscales are reported, in others selected subscales and in two, [25,28] no subscales are reported. Such selective reporting might increase the likelihood of presentation of findings supporting a favored hypothesis and the omission of less favorable analyses. Before- and after- data from the intervention group were usually presented more prominently than between-group comparisons, and this often obscured interpretation of group effects. In the 33 papers tabulated above, all except one [29] report at least one statistically significant positive child-based outcome for Triple P compared to the control condition in the Results sections, while 25/33 papers report at least one statistically nonsignificant result. Only 4/33 abstracts report any negative findings, whereas $32 / 33$ report positive findings so that abstracts tend to give a more favorable picture of the effects of Triple P interventions than are supported by the more detailed findings.

\section{Risk of bias within studies - whole-population ('public health') interventions}

Three whole-population studies met our inclusion criteria. The South Carolina study [30] was a well-designed cluster randomized trial, but the presentation did not comply with the recommended Consolidated Standards for Reporting Trials (CONSORT) format for the reporting of 
Table 1 Risk of bias in individual studies.

\begin{tabular}{|c|c|c|c|c|c|c|c|}
\hline Paper & $\begin{array}{l}\text { Blinding of } \\
\text { assessors? }\end{array}$ & $\begin{array}{l}\text { Treatment and control } \\
\text { groups similar at } \\
\text { baseline? }\end{array}$ & $\begin{array}{l}\text { Percentage } \\
\text { drop out at } \\
\text { post } \\
\text { intervention } \\
\text { measure? }\end{array}$ & $\begin{array}{l}\text { Analyzed by } \\
\text { intention to } \\
\text { treat }\end{array}$ & $\begin{array}{l}\text { Subgroup } \\
\text { analyses } \\
\text { reported? }\end{array}$ & $\begin{array}{l}\text { Statement of study } \\
\text { funding }\end{array}$ & $\begin{array}{l}\text { Included in } \\
\text { meta-analysis? }\end{array}$ \\
\hline $\begin{array}{l}\text { Bodenmann } \\
\text { et al. [32] }\end{array}$ & No & Yes & $\begin{array}{l}\text { Triple P 5\% } \\
\text { CCET 8\% } \\
\text { Control 23\% } \\
\text { (at long term } \\
\text { follow up) }\end{array}$ & No & Yes & $\begin{array}{l}\text { Yes. Gebert Ruef } \\
\text { Foundation } \\
\text { (Switzerland) }\end{array}$ & Yes \\
\hline $\begin{array}{l}\text { Connell et al. } \\
\text { [50] }\end{array}$ & No & $\begin{array}{l}\text { More females in control } \\
\text { group }\end{array}$ & $\begin{array}{l}\text { Intervention } \\
0 \% \\
\text { Control } 8 \%\end{array}$ & No & Yes & No & Yes \\
\hline $\begin{array}{l}\text { Gallart \& } \\
\text { Matthey [26] }\end{array}$ & No & Yes (not tabulated) & $\begin{array}{l}\text { Not stated } \\
(9 \% \text { overall) }\end{array}$ & No & Yes & No & Yes \\
\hline $\begin{array}{l}\text { Hahlweg et } \\
\text { al. [51] }\end{array}$ & No & Yes & $\begin{array}{l}\text { Intervention } \\
\text { mothers 14\% } \\
\text { Control } \\
\text { mothers 3\% } \\
\text { All fathers } \\
\text { 19\% (unable } \\
\text { to distinguish } \\
\text { intervention } \\
\text { \& control } \\
\text { attrition) }\end{array}$ & No & No & $\begin{array}{l}\text { Yes. Deutsche } \\
\text { Forschungsgemeinschaft }\end{array}$ & Yes \\
\hline $\begin{array}{l}\text { Hahlweg et } \\
\text { al. [13] (data } \\
\text { also reported } \\
\text { in [52]) }\end{array}$ & $\begin{array}{l}\text { No (parents } \\
\text { and } \\
\text { teachers) } \\
\text { Yes } \\
\text { (observers) }\end{array}$ & $\begin{array}{l}\text { More parents in control } \\
\text { group were single in } \\
\text { comparison to the } \\
\text { intervention group: } 34 \% \\
\text { and } 15.6 \% \text {, respectively. } \\
\text { Baseline differences } \\
\text { between groups for two- } \\
\text { parent households }\end{array}$ & $\begin{array}{l}\text { Intervention } \\
0.5 \% \\
\text { Control } 1 \%\end{array}$ & Yes & Yes & $\begin{array}{l}\text { Yes. Deutsche } \\
\text { Forschungsgemeinschaft }\end{array}$ & Yes \\
\hline $\begin{array}{l}\text { Hoath \& } \\
\text { Sanders [53] }\end{array}$ & $\begin{array}{l}\text { No (parents) } \\
\text { Not known } \\
\text { (teachers) }\end{array}$ & $\begin{array}{l}\text { Control group had lower } \\
\text { family income }\end{array}$ & $\begin{array}{l}\text { Intervention } \\
10 \% \\
\text { Control } 0 \%\end{array}$ & No & No & No & Yes \\
\hline $\begin{array}{l}\text { Joachim et al. } \\
\text { [54] }\end{array}$ & No & $\begin{array}{l}\text { Higher proportion of male } \\
\text { children in control group }\end{array}$ & $\begin{array}{l}\text { Intervention } \\
15 \% \\
\text { Control } 10 \%\end{array}$ & Yes & Yes & No & Yes \\
\hline $\begin{array}{l}\text { Leung et al. } \\
{[55]}\end{array}$ & No & Yes & $\begin{array}{l}\text { Intervention } \\
28 \% \\
\text { Control } 20 \%\end{array}$ & $\begin{array}{l}\text { Yes - but only } \\
\text { per protocol } \\
\text { results } \\
\text { tabulated }\end{array}$ & No & No & Yes \\
\hline $\begin{array}{l}\text { Markie-Dadds } \\
\text { \& Sanders } \\
\text { [56] }\end{array}$ & No & Yes & $\begin{array}{l}\text { Intervention } \\
3 \% \\
\text { Control } 0 \%\end{array}$ & No & Yes & No & Yes \\
\hline $\begin{array}{l}\text { Markie-Dadds } \\
\text { \& Sanders } \\
\text { [57] }\end{array}$ & No & Yes & $\begin{array}{l}\text { Intervention } \\
28 \% \\
\text { Control 23\% }\end{array}$ & $\begin{array}{l}\text { Yes - but only } \\
\text { per protocol } \\
\text { results } \\
\text { tabulated }\end{array}$ & Yes & $\begin{array}{l}\text { Queensland Health and } \\
\text { the National Health and } \\
\text { Medical Research } \\
\text { Council }\end{array}$ & Yes \\
\hline $\begin{array}{l}\text { Martin \& } \\
\text { Sanders [58] }\end{array}$ & No & $\begin{array}{l}\text { Treatment group had lower } \\
\text { ECBI scores }\end{array}$ & $\begin{array}{l}\text { Intervention } \\
30 \% \\
\text { Control 50\% }\end{array}$ & No & Yes & No & Yes \\
\hline $\begin{array}{l}\text { Matsumoto } \\
\text { et al. [59] }\end{array}$ & No & Yes & $\begin{array}{l}\text { Intervention } \\
0 \% \\
\text { Control 0\% }\end{array}$ & Yes (in effect) & No & No & Yes \\
\hline $\begin{array}{l}\text { Matsumoto } \\
\text { et al. [60] }\end{array}$ & No & $\begin{array}{l}\text { No. ECBI scores substantially } \\
\text { higher in intervention group }\end{array}$ & Not stated & No & No & No & Yes \\
\hline $\begin{array}{l}\text { McTaggart \& } \\
\text { Sanders [21] }\end{array}$ & No & Yes & Not known & No & Yes & No & Not ECBI/CBCL \\
\hline $\begin{array}{l}\text { Morawska \& } \\
\text { Sanders [61] }\end{array}$ & $\begin{array}{l}\text { No (parents) } \\
\text { Yes } \\
\text { (observers) }\end{array}$ & Yes & $\begin{array}{l}\text { Intervention } \\
12 \% \\
\text { Control } 10 \%\end{array}$ & $\begin{array}{l}\text { Yes - but only } \\
\text { per protocol } \\
\text { results } \\
\text { tabulated }\end{array}$ & Yes & No & Yes \\
\hline
\end{tabular}


Table 1 Risk of bias in individual studies. (Continued)

\begin{tabular}{|c|c|c|c|c|c|c|c|}
\hline $\begin{array}{l}\text { Morawska \& } \\
\text { Sanders [62] }\end{array}$ & No & $\begin{array}{l}\text { No. ECBI scores substantially } \\
\text { higher in intervention group }\end{array}$ & $\begin{array}{l}\text { Intervention } \\
11 \% \\
\text { Control } 3 \%\end{array}$ & $\begin{array}{l}\text { Yes - but only } \\
\text { per protocol } \\
\text { results } \\
\text { tabulated }\end{array}$ & Yes & $\begin{array}{l}\text { Yes. Telstra } \\
\text { Foundation. }\end{array}$ & Yes \\
\hline $\begin{array}{l}\text { Morawska et } \\
\text { al. [63] }\end{array}$ & No & Yes & $\begin{array}{l}\text { Intervention } \\
18 \% \\
\text { Control 18\% }\end{array}$ & $\begin{array}{l}\text { Yes - but only } \\
\text { per protocol } \\
\text { results } \\
\text { tabulated }\end{array}$ & Yes & No & Yes \\
\hline $\begin{array}{l}\text { Nicholson \& } \\
\text { Sanders [28] }\end{array}$ & $\begin{array}{l}\text { No (parents } \\
\text { and step } \\
\text { parents), } \\
\text { Possibly } \\
\text { (teenager's } \\
\text { self-report) }\end{array}$ & Yes & $\begin{array}{l}40 \% \\
\text { therapist- } \\
\text { delivered } \\
45 \% \text { self- } \\
\text { delivered } \\
5 \% \text { waiting } \\
\text { list control }\end{array}$ & No & yes & $\begin{array}{l}\text { Yes. National Health and } \\
\text { Medical Research } \\
\text { Council }\end{array}$ & Not ECBI/CBCL \\
\hline $\begin{array}{l}\text { Plant \& } \\
\text { Sanders [64] }\end{array}$ & $\begin{array}{l}\text { Yes (video } \\
\text { observations) } \\
\text { No (parent } \\
\text { report) }\end{array}$ & Yes & $\begin{array}{l}\text { Nil in all } \\
\text { three groups }\end{array}$ & Yes (in effect) & Yes & $\begin{array}{l}\text { Yes. Australian Research } \\
\text { Council and Apex } \\
\text { Foundation }\end{array}$ & $\begin{array}{l}\text { ECBI only used } \\
\text { as entry } \\
\text { screener }\end{array}$ \\
\hline Prinz et al. [5] & Not clear & $\begin{array}{l}\text { Not clear (five year average } \\
\text { data presented) }\end{array}$ & Not known & Yes (in effect) & No & Yes. US CDC & Not ECBI/CBCL \\
\hline $\begin{array}{l}\text { Roberts et al. } \\
\text { [33] }\end{array}$ & $\begin{array}{l}\text { Yes (video } \\
\text { observations) } \\
\text { No (parent } \\
\text { report) }\end{array}$ & In some scales & $\begin{array}{l}37 \% \\
\text { intervention } \\
35 \% \text { control }\end{array}$ & No & Yes & $\begin{array}{l}\text { Yes. Western Australian } \\
\text { Health Promotion } \\
\text { Foundation }\end{array}$ & Not ECBI/CBCL \\
\hline $\begin{array}{l}\text { Sanders et al. } \\
\text { [65] }\end{array}$ & $\begin{array}{l}\text { Yes (video } \\
\text { observations) } \\
\text { No (parent } \\
\text { report) }\end{array}$ & No data presented & $\begin{array}{l}\text { EBFI 23\%; } \\
\text { SBFI } 17 \% \\
\text { SDBFI } 18 \% \\
\text { control } 8 \%\end{array}$ & No & Yes & $\begin{array}{l}\text { Yes. Grants from } \\
\text { Queensland Health and } \\
\text { the National Health and } \\
\text { Medical Research } \\
\text { Council }\end{array}$ & Yes \\
\hline $\begin{array}{l}\text { Sanders et al. } \\
{[66]}\end{array}$ & No & Yes & Not stated & Not clear & Yes & $\begin{array}{l}\text { Partial - acknowledged } \\
\text { source of TV programs } \\
\text { and funding for } \\
\text { distribution of video } \\
\text { material }\end{array}$ & Yes \\
\hline $\begin{array}{l}\text { Sanders et al. } \\
\text { [6] }\end{array}$ & No & $\begin{array}{l}\text { No. Intervention area } \\
\text { sample younger, poorer, less } \\
\text { well educated and more } \\
\text { likely to be single }\end{array}$ & $\begin{array}{l}\text { Not } \\
\text { applicable }\end{array}$ & Not applicable & Yes & Yes. Several funders & Not ECBI/CBCL \\
\hline $\begin{array}{l}\text { Sanders et al. } \\
\text { [27] }\end{array}$ & No & $\begin{array}{l}\text { No data presented except } \\
\text { baseline measures }\end{array}$ & $\begin{array}{l}\text { Intervention } \\
23 \% \\
\text { Control } 12 \%\end{array}$ & Yes & No & $\begin{array}{l}\text { Yes. Australia Research } \\
\text { Council }\end{array}$ & Yes \\
\hline $\begin{array}{l}\text { Stallman \& } \\
\text { Ralph [25] }\end{array}$ & $\begin{array}{l}\text { No (parents) } \\
\text { Possibly } \\
\text { (teenager's } \\
\text { self-report) }\end{array}$ & Yes & $\begin{array}{l}\text { Intervention } \\
19 \% \\
\text { Control 11\% }\end{array}$ & $\begin{array}{l}\text { Yes, but only } \\
\text { per protocol } \\
\text { results } \\
\text { tabulated }\end{array}$ & Yes & $\begin{array}{l}\text { Yes. Australian Rotary } \\
\text { Health Research Fund, } \\
\text { grant }\end{array}$ & Not ECBI/CBCL \\
\hline $\begin{array}{l}\text { Turner et al. } \\
\text { [67] }\end{array}$ & No & Yes & $\begin{array}{l}\text { Intervention } \\
23 \% \\
\text { Control } 28 \%\end{array}$ & No & Yes & $\begin{array}{l}\text { Yes. Queensland Health } \\
\text { and Queensland } \\
\text { Department of Premier } \\
\text { and Cabinet }\end{array}$ & Yes \\
\hline $\begin{array}{l}\text { Turner \& } \\
\text { Sanders [68] }\end{array}$ & $\begin{array}{l}\text { Yes (video } \\
\text { observations) } \\
\text { No (parent } \\
\text { report) }\end{array}$ & Yes & $\begin{array}{l}\text { Intervention } \\
19 \% \\
\text { Control 14\% }\end{array}$ & $\begin{array}{l}\text { For measures } \\
\text { with a } \\
\text { significant } \\
\text { univariate } \\
\text { condition } \\
\text { effect at post- } \\
\text { assessment }\end{array}$ & Yes & No & Yes \\
\hline $\begin{array}{l}\text { Turner et al. } \\
\text { [29] }\end{array}$ & $\begin{array}{l}\text { Yes (video } \\
\text { observations) } \\
\text { No (parent } \\
\text { report) }\end{array}$ & Yes & $\begin{array}{l}\text { Intervention } \\
0 \% \\
\text { Control 11\% }\end{array}$ & No & Yes & $\begin{array}{l}\text { Yes. National Health and } \\
\text { Medical Research } \\
\text { Council of Australia }\end{array}$ & Not ECBI/CBCL \\
\hline $\begin{array}{l}\text { West et al. } \\
\text { [22] }\end{array}$ & No & Yes & $\begin{array}{l}\text { Intervention } \\
21 \% \\
\text { Control } 6 \%\end{array}$ & Yes & Yes & $\begin{array}{l}\text { Yes. Telstra } \\
\text { Foundation }\end{array}$ & Not ECBI/CBCL \\
\hline $\begin{array}{l}\text { Whittingham } \\
\text { et al. [24] }\end{array}$ & No & Yes & $\begin{array}{l}\text { Intervention } \\
0 \% \\
\text { Control 10\% }\end{array}$ & Yes & Yes & $\begin{array}{l}\text { Yes. School of } \\
\text { Psychology University of } \\
\text { Queensland }\end{array}$ & Yes \\
\hline
\end{tabular}


Table 1 Risk of bias in individual studies. (Continued)

\begin{tabular}{|c|c|c|c|c|c|c|c|}
\hline $\begin{array}{l}\text { Wiggins et al. } \\
\text { [23] }\end{array}$ & No & Yes & $\begin{array}{l}\text { Intervention } \\
10 \% \\
\text { Control 26\% }\end{array}$ & Yes & Yes & No & Yes \\
\hline $\begin{array}{l}\text { Zubrick et al. } \\
{[20]}\end{array}$ & No & $\begin{array}{l}\text { No. Intervention area } \\
\text { sample had younger } \\
\text { children, less highly } \\
\text { educated parents, more } \\
\text { parenting problems and } \\
\text { higher child ECBI scores. } \\
\text { Different recruitment } \\
\text { methods in intervention } \\
\text { and control areas }\end{array}$ & $\begin{array}{l}\text { Intervention } \\
14 \% \\
\text { Control } 4 \%\end{array}$ & Not applicable & Yes & $\begin{array}{l}\text { Yes. Western Australian } \\
\text { Department of Health }\end{array}$ & $\begin{array}{l}\text { No - Not } \\
\text { randomized, } \\
\text { and } \\
\text { uncorrected } \\
\text { outcome data } \\
\text { for control } \\
\text { group not } \\
\text { given }\end{array}$ \\
\hline
\end{tabular}

CBCL, Child Behavior Checklist; CCET, Couples Coping Enhancement Training; EBCl, Eyberg Child Behavior Inventory; EBFI, Enhanced Behavioural Family Intervention (level 5); SBFI, Standard Behavioural Family Intervention (level 4); SDBFI, Self-directed Behavioural Family Intervention (level 4).

cluster randomized trials [31], making an accurate assessment of the implications of the paper difficult. Although it claimed to have achieved a reduction in the incidence of episodes of child maltreatment [5], it actually demonstrated an unexplained rise in reports in control areas rather than a drop in Triple $\mathrm{P}$ intervention sites. The description of the random allocation was poor, and the analysis was simplistic, being a two-sample t-test of county-wide measures. In particular, although some form of stratification or matching was used (it was not clear exactly how this had been done), there was no evidence that this had been accounted for in the analysis. For example, if counties were randomized within pairs, then the within-pair differences in the changes from baseline would have been of interest, but these were not reported. Therefore, although there are positive conclusions from this study, some doubt remains as to their validity.

There are two other whole-population Triple P evaluations involving a comparison group. Sanders et al. [6] reported a quasi-experimental study in parts of Brisbane, Sydney and Melbourne. There were substantial baseline differences between intervention and control populations. Approximately 3,000 parents were interviewed before and after the intervention, but different samples were used in each data collection and so it is not possible to characterize changes in individuals over time. Results are reported only as proportion of children with 'clinically elevated' scores rather than mean or median results. The positive child-based outcomes reported with this approach, from seven possible outcomes, were in the emotional and total problems domains of the Strengths and Difficulties Questionnaire, although neither finding would have attained conventional levels of statistical significance had allowance for multiple comparisons been made. We consider that this study offers relatively little support for any effect of triple P on children at the whole-population level.

Zubrick et al. [20] reported a further quasi-experimental study in two areas of Western Australia. There were again substantial differences in the characteristics of intervention and control populations. Recruitment methods differed significantly between the two areas: in the intervention area parents volunteered for active participation whereas in the control area parents volunteered to take part in a health services survey of child behavior. Analysis using hierarchical linear modelling suggests a short term improvement in ECBI externalizing behavior scores but given the potential for confounding by factors such as parental motivation it is difficult to confirm that this difference is attributable to the intervention.

\section{Results of individual studies}

The 23 papers listed in Table 2 and associated data were used in the meta-analysis. These papers report the randomized trials in which the principal carer (usually the mother) of the index child returned ECBI or CBCL data before and after the intervention. Insufficient information is presented in most publications to allow use of intention-to-treat data, so non-imputed data for study completers only are used here.

Only two studies [29,32] compared a Triple-P intervention with an active comparison condition - a marital distress prevention program (Couples Coping Enhancement Training, $\mathrm{n}=50$ per group) [32] and standard dietary education (group $n=12$ and 9) [29]. No significant differences between the active intervention groups in terms of maternal or paternal reports of child behavior were reported in either study.

The whole population studies were excluded from the meta-analysis on the grounds of non-randomized design or the nature of the reported outcome measures.

\section{Synthesis of results}

The forest plot (Hedges) depicting the included studies (maternal report ECBI-I or CBCL-E) is shown in Figure 2.

For the (generally) maternally-reported ECBI-I and CBCL-E, the summary ES was 0.61 (95\%CI 0.42, 0.79) 
Table 2 Papers included in the meta-analysis.

\begin{tabular}{|c|c|c|c|c|c|c|c|}
\hline \multirow[b]{2}{*}{ Author } & \multirow{2}{*}{$\begin{array}{c}\text { Pre-intervention } \\
\text { Pooled baseline score }\end{array}$} & \multirow[b]{2}{*}{$\mathrm{n} 1$} & \multicolumn{4}{|c|}{ Post-intervention } & \multirow[b]{2}{*}{ sd2 } \\
\hline & & & mean1 & sd1 & $\mathrm{n} 2$ & mean2 & \\
\hline Bodenmann et al. [32] ${ }^{\mathrm{a}}$ & 118.1 & 50 & 115.4 & 22.6 & 50 & 104.7 & 23.9 \\
\hline Connell et al. [50] & 157.0 & 12 & 159 & 10.58 & 12 & 117.33 & 22.77 \\
\hline Gallart \& Matthey [26] & N/A & 17 & 137.1 & 34.8 & 17 & 112 & 31.7 \\
\hline Hahlweg et al. [51]* & 13.2 & 31 & 13 & 7.6 & 32 & 7.8 & 5.7 \\
\hline Hahlweg et al. [13]* & 11.6 & 57 & 9.3 & 6.6 & 169 & 10.43 & 7.43 \\
\hline Hoath \& Sanders [53] & 162.1 & 11 & 148.36 & 40.29 & 9 & 125.22 & 35.63 \\
\hline Joachim et al. [54] & 129.5 & 18 & 130.17 & 27.75 & 22 & 109.41 & 27.36 \\
\hline Leung et al. [55] & 134.7 & 36 & 136.45 & 27.3 & 33 & 107.28 & 31.03 \\
\hline Markie-Dadds \& Sanders [56] & 148.6 & 12 & 146.92 & 15.53 & 28 & 116.3 & 31.53 \\
\hline Markie-Dadds \& Sanders [57] & 132.7 & 22 & 136.23 & 31.62 & 21 & 100.76 & 29.9 \\
\hline Martin \& Sanders [58] & 130.3 & 11 & 126.09 & 28.11 & 16 & 99.88 & 22.39 \\
\hline Matsumoto et al. [59] & 106.3 & 25 & 105.8 & 25.28 & 25 & 94.12 & 23.79 \\
\hline Matsumoto et al. [60] & 112.7 & 26 & 107.04 & 29.25 & 25 & 104.12 & 24.45 \\
\hline Morawska \& Sanders [61] & 120.7 & 37 & 123.4 & 27.54 & 75 & 108.59 & 22.96 \\
\hline Morawska \& Sanders [62] & 118.1 & 34 & 111.71 & 28.8 & 32 & 103.38 & 25.67 \\
\hline Morawska et al. [63] & 146.8 & 27 & 152.26 & 27.14 & 23 & 124.7 & 20.61 \\
\hline Sanders et al. [65] & 152.8 & 71 & 136.79 & 28.42 & 184 & 113.32 & 29.53 \\
\hline Sanders et al. [66] & 115.9 & 28 & 108.59 & 33.36 & 28 & 98.74 & 28.04 \\
\hline Sanders et al. [27] & 121.7 & 40 & 119.31 & 25.8 & 33 & 111.77 & 30.87 \\
\hline Turner \& Sanders [68] & 128.8 & 13 & 112.25 & 20.50 & 12 & 114.08 & 22.69 \\
\hline Turner et al. [67] & 140.6 & 18 & 130.74 & 33.97 & 20 & 124.14 & 31.71 \\
\hline Whittingham et al. [24] & 143.1 & 30 & 148.63 & 30.33 & 29 & 121.4 & 25.28 \\
\hline Wiggins et al. [23]* & 65.1 & 22 & 63.4 & 10.4 & 27 & 57.7 & 9.7 \\
\hline
\end{tabular}

Pooled mean pre-intervention score and post-intervention scores: $\mathrm{n} 1$, mean 1 and sd1 are respectively group size, mean and standard deviation for the control groups post-intervention, and $\mathrm{n} 2$, mean 2 and $\mathrm{sd} 2$ are the corresponding figures for the Triple-P intervention groups. Means and standard deviations are for ECBII subscale data, apart from the three papers marked with an asterisk, where the CBCL-E was reported. ${ }^{a} E C B I$ subscale data reported in [32] were assumed to have been transposed, and are corrected here. In this paper attrition rates at the post-treatment assessment are unknown and we assumed they remained constant. CBCL-E, Child Behavior Checklist - Externalizing Scale; ECBI-I, Eyberg Child Behavior Inventory - Intensity scale.

under the definition of a random-effects model with Hedges correction. Thirteen of the studies showed a significant positive effect while ten did not, with most ESs falling in the range 0.3 to 1.0 .

There is evidence of heterogeneity (chi-squared $=$ 60.16 (d.f. $=22) P=0.000$ ), with the variation in SMD attributable to heterogeneity (I-squared) $=63.4 \%$. This level of heterogeneity indicates that there are significant differences between the studies which cannot be explained by random variation.

\section{Risk of bias across studies}

Publication bias was assessed by the use of a funnel plot, which illustrates the relationship between sample size and ES. Publication bias is present when there is selective reporting of small studies with positive results. Larger studies are more likely to be published successfully regardless of ES. The results are shown in Figure 3.

Egger's test (regression of the standard normal deviate of intervention effect estimate against its standard error) yielded limited evidence of small-study effects $(P=$ 0.067), with an estimated bias coefficient of 1.98 .

\section{Additional analysis}

Sensitivity analysis in relation to author affiliation was not possible because of the small number of articles published without Triple-P affiliated authorship.

The meta-regression comparing baseline severity scores with ES for those studies which employed the ECBI-I outcome is shown in Figure 4. Ninety four percent of the between-study variance is explained by the covariate mean baseline value, and a ten-point increase in baseline ECBI-I score is associated with a 0.15 increase in ES (95\% CI: $0.005,0.025)$. The mean baseline ECBI-I score was 132.7, and the ES for the studies included in this metaregression was 0.65 .

Summaries of child-based outcomes reported by informants other than the principal (usually maternal) carer are reported in Table 3.

Independent observers reported benefit attributable to Triple P on at least one subscale of an observational measure in two of seven papers in which these data are reported. Teachers reported benefit in one subscale score in one of four papers with relevant data. Seven papers yielded data on paternally reported ECBI-I or CBCL-E. Summary data are reported in Table 4. 


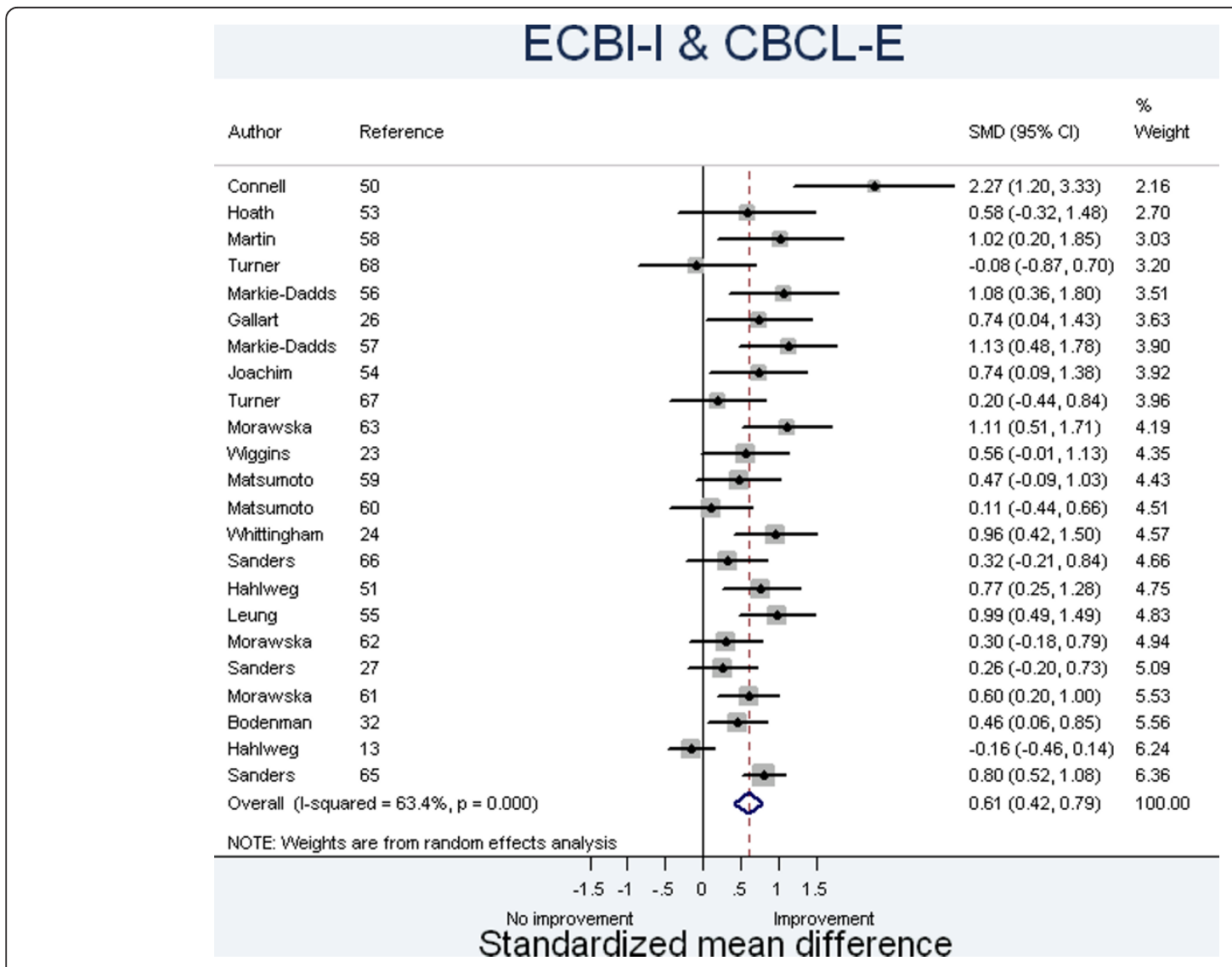

Figure 2 Forest Plot (Hedges) of Standardized Mean Differences. Studies reporting data based on ECBI or CBCL questionnaires completed by mothers are presented in increasing order of weight to final estimate, based on sample size. CBCL, Child Behavior Checklist; ECBI, Eyberg Child Behavior Inventory.

There was strong evidence of heterogeneity (chi-squared $=29.72$ (d.f. = 5) $P<0.001$ ), with a variation in SMD attributable to heterogeneity (I-squared) of $83 \%$. The summary

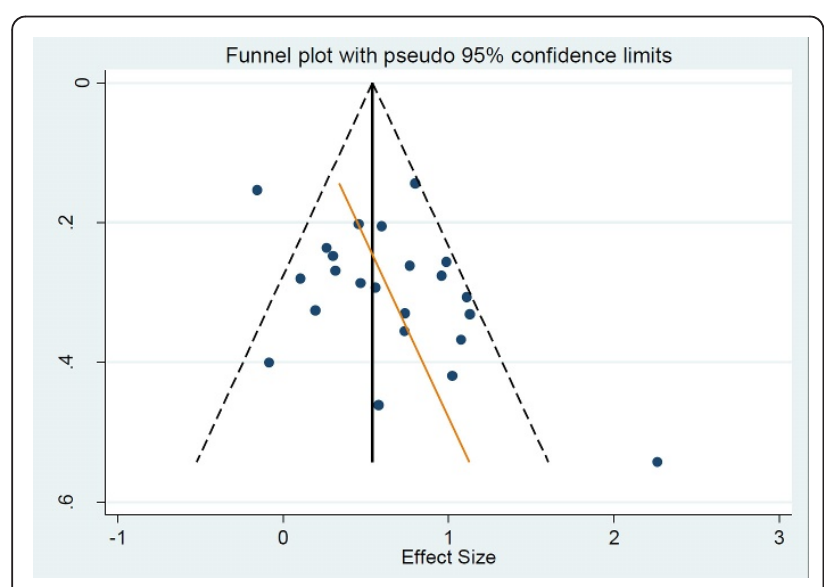

Figure 3 Funnel plots for the random effects model (Hedges) based on maternally-reported ECBI-I or CBCL-E data. CBCL, Child Behavior Checklist; ECBI-I, Eyberg Child Behavior Inventory Intensity scale.
ES for the six studies for which data were presented was $0.42(95 \% \mathrm{CI}-0.02,0.87)$ under the definition of a randomeffects model. The remaining study reporting non-significant results could have influenced this estimate in either direction.

\section{Discussion}

There are a large number of published evaluations of Triple-P parenting interventions and we were able to identify 33 English-language studies which measured a child-based outcome and which compared Triple P interventions with a comparison condition. Most of the studies involved families who responded to media advertisements. These families clearly have children whose parents are finding difficulties with their behavior but may well not be typical of such families in the population. They are more likely to be motivated and literate and are sufficiently confident to present for treatment as volunteers. These characteristics would be likely to lead to high levels of compliance with treatment and better than average treatment response. Only five studies [5,6,21,33,34] did not rely upon self-referral by potential participants. All the studies 


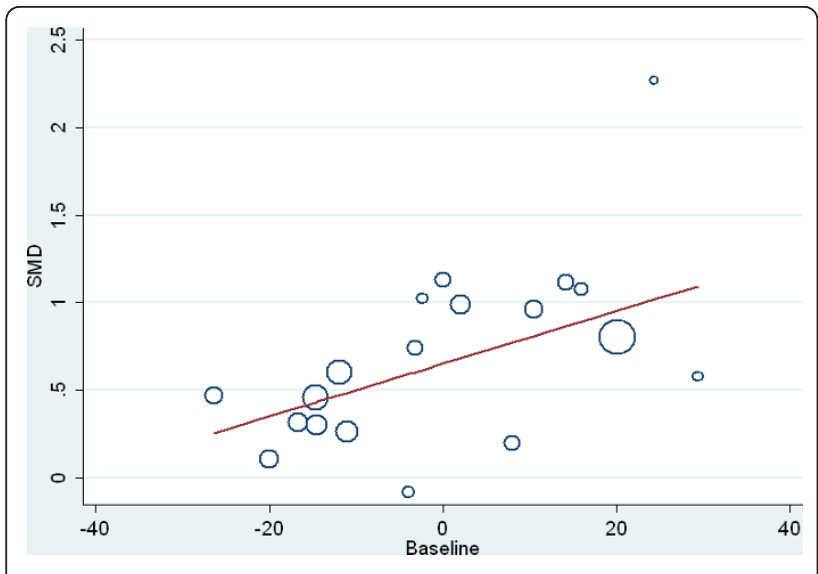

Figure 4 Bubble plot of standardized between-group mean difference (SMD - equivalent to effect size) against preintervention (baseline) pooled ECBI-I scores. The baseline ECBI-I scores are centered on the mean value across all included studies. The size of the circle represents the study sample size. ECBI-I, Eyberg Child Behavior Inventory - Intensity scale.

involved only children over two years old. There are many forms of the Triple-P program [2], and new versions emerge regularly, but for simplicity we have not distinguished between the levels of intervention. Nevertheless, most of the studies reported on the effectiveness of small group-based Triple-P interventions, usually level 4 or 5 , and synthesis of results from other Triple P levels would be limited by low numbers.

Most of the studies were relatively small and the great majority used a waiting-list control design in which the participants on the waiting list were offered active treatment immediately after the post-intervention data collection. It is, therefore, not possible to draw conclusions about the longer term effectiveness of Triple P relative to a comparison condition. Before- and after- data from the intervention group were usually presented more prominently (and frequently) than between-group comparisons, and this method of reporting often obscured interpretation of group effects and tended to increase the impression of a positive effect from the intervention. Only two trials used an active comparison group, and neither of these showed any advantage for Triple $\mathrm{P}$ in terms of child-based outcomes. Trials with waiting list controls or usual care provide intervention estimates which reflect the combined specific and non-specific effects that will accrue in practice, and are more likely to show betweengroup differences than trials with active controls [35].

A range of child-based outcome measures was used but the most commonly reported was the ECBI, completed by parents. In the majority of cases (31/33) the main informant was the mother, and these data were synthesized in our main meta-analysis. Despite some differences in methodological approach, the ES obtained in our meta-analysis of maternally-reported ECBI scores (0.61) is impressive and is broadly in line with that reported by other authors $[9,11,12]$.

There is some evidence of publication bias from our analyses of the published work and there is additional evidence that results of a number of evaluations of Triple-P have not been published [30,36]. Further evaluation of publication bias is not possible given the uniform lack of Triple-P trial registration. Thus, despite the apparent consistency of more recently published work, there is still the possibility that these studies represent a particularly favorable picture of Triple-P. The International Committee of Medical Journal Editors (ICMJE) agreed in 2005 that only registered trials would be considered for publication. Allowing for publication time lags, about one third of the studies in our meta-analysis pre-dated the guidance and two thirds could potentially have benefitted from adopting these recommendations.

There are considerable limitations in relying on maternal report data alone: 17 papers gave outcomes reported by informants other than mothers. Five of these studies showed a relative benefit for Triple P on one or more outcome measures compared with no treatment. Meta-analytic synthesis of paternally-reported data identified a pooled ES of 0.42 but there was significant heterogeneity and the overall effect size was not significantly greater than zero. Multiple outcomes were used in five of the six papers reporting significantly positive results: primary outcomes were not pre-specified (in common with all the reported trials), and corrections were not made for multiple comparisons. Paternal reports are often difficult to assess because of missing data which may not be missing at random. The incorporation of independent direct observations of parent and child behavior into trial design provides important confirmatory information [37,38], and seven of the Triple P trials (see Table 4) included data from independent observers. Two of these seven studies reported benefit in one or more observational subscale.

It is possible that the discrepancy between maternal and paternal (or independent) reports of child behavior may be accounted for by the fact that maternal mental state improved significantly with most Triple-P interventions [10] and this may have led to a more positive maternal evaluation of the child's behavior, reflecting more optimistic states of mind. Fathers are less likely to attend sessions and independent observers are unconnected with the intervention. Related attribution effects have been reported in relation to Triple-P [39]. It is also possible that mothers are more accurate than fathers in reporting their children's behavior difficulties. One paper [13] reported a planned subgroup analysis for lone parent families - and reported no benefit from the triple P intervention.

All of the papers considered here, with one exception [26] were authored or influenced by Triple-P affiliated personnel. This is commonly observed in the early stages 
Table 3 Child based outcomes reported by informants other than the child's mother.

\begin{tabular}{|c|c|c|c|c|}
\hline Paper & $\begin{array}{l}\text { Number of } \\
\text { children/ } \\
\text { informants }\end{array}$ & Informant & Nature of child-based outcome measure(s) & $\begin{array}{l}\text { Significance }(P<0.05) \text { of improvement } \\
\text { with intervention versu control }\end{array}$ \\
\hline $\begin{array}{l}\text { Bodenmann et } \\
\text { al. [32] }\end{array}$ & 150 & Father & $\mathrm{ECBI}$ & $\begin{array}{l}\text { Not significant (Intensity and Problem } \\
\text { subscales) }\end{array}$ \\
\hline \multirow[t]{2}{*}{$\begin{array}{l}\text { Connell et al. } \\
\text { [50] }\end{array}$} & 23 & Father & $\mathrm{ECBI}$ & $\begin{array}{l}\text { Significant benefit (Intensity and Problem } \\
\text { subscales) }\end{array}$ \\
\hline & & Father & PDRC - Parent diary record checklist & Not significant \\
\hline \multirow[t]{2}{*}{$\begin{array}{l}\text { Hahlweg et al. } \\
\text { [51] }\end{array}$} & 43 & Father & $\begin{array}{l}\text { Child Behavior Checklist-Parent Report (CBCL 11/2-5, } \\
\text { German version) }\end{array}$ & Not significant \\
\hline & & Father & SDQ & Not significant \\
\hline \multirow{3}{*}{$\begin{array}{l}\text { Hahlweg et al. } \\
\text { [13] }\end{array}$} & 198 & Father & $\mathrm{CBCL}$ & Not significant \\
\hline & 273 & Observers & Revised Family Observation Schedule (FOS-RIII). & Not significant \\
\hline & 177 & Teachers & Caregiver Teacher Report Form (C-TRF 1.5 - 5) & Not significant \\
\hline \multirow[t]{2}{*}{$\begin{array}{l}\text { Hoath \& } \\
\text { Sanders [53] }\end{array}$} & 21 & Teachers & $\begin{array}{l}\text { Sutter-Eyberg Student Behavior Inventory-Revised } \\
\text { (SESBI-R) }\end{array}$ & Not significant \\
\hline & & Teachers & Child Attention Problems Rating Scale (CAP) & Not significant \\
\hline \multirow{2}{*}{$\begin{array}{l}\text { Markie-Dadds \& } \\
\text { Sanders [56] }\end{array}$} & & Father & $\mathrm{ECBI}$ & Not significant \\
\hline & & Father & Parent Daily Report & Not significant \\
\hline $\begin{array}{l}\text { Morawska \& } \\
\text { Sanders [62] }\end{array}$ & 75 & Teacher & Strengths and Difficulties Questionnaire & $\begin{array}{l}\text { Not significant apart from hyperactivity } \\
\text { subscale }\end{array}$ \\
\hline $\begin{array}{l}\text { McTaggart \& } \\
\text { Sanders [21] }\end{array}$ & & Teacher & Sutter-Eyberg Student Behavior Inventory (SESBI) & $\begin{array}{l}\text { Not significant (except when baseline } \\
\text { adjustment used) }\end{array}$ \\
\hline \multirow{2}{*}{$\begin{array}{l}\text { Morawska \& } \\
\text { Sanders [61] }\end{array}$} & 73 & Father & $\mathrm{ECBI}$ & Not significant \\
\hline & & Observers & Family observation schedule & Not significant \\
\hline \multirow{3}{*}{$\begin{array}{l}\text { Nicholson \& } \\
\text { Sanders [28] }\end{array}$} & 42 & Self report & Child Depression Inventory & Not significant \\
\hline & & Self report & Child Manifest Anxiety Scale & Not significant \\
\hline & & Self report & Coopersmith Self-Esteem Inventory & Not significant \\
\hline $\begin{array}{l}\text { Plant \& Sanders } \\
\text { [64] }\end{array}$ & 74 & $\begin{array}{l}\text { Independent } \\
\text { observers }\end{array}$ & Revised Family Observation Schedule (FOS-RIII). & $\begin{array}{l}\text { Significant benefit on negative behavior } \\
\text { subscales (other subscales not reported) }\end{array}$ \\
\hline \multirow[t]{4}{*}{ Prinz et al. [5] } & $\begin{array}{l}\text { Approximately } \\
170,000\end{array}$ & $\begin{array}{l}\text { Child } \\
\text { Protective } \\
\text { Services }\end{array}$ & Numbers of substantiated child maltreatment cases & Significant benefit ${ }^{a}$ \\
\hline & & $\begin{array}{l}\text { Foster Care } \\
\text { System }\end{array}$ & Out of home placements & Significant benefit ${ }^{a}$ \\
\hline & & Hospitals & Hospital visits for maltreatment & Significant benefit ${ }^{a}$ \\
\hline & & $\begin{array}{l}\text { Child } \\
\text { Protective } \\
\text { Services }\end{array}$ & Maltreatment investigation & Not significant \\
\hline \multirow[t]{7}{*}{$\begin{array}{l}\text { Roberts et al. } \\
\text { [33] }\end{array}$} & 23 & Father & $\begin{array}{l}\text { Total behavior problem subscale score of } \\
\text { Developmental Behavior Checklist Parent Version. }\end{array}$ & Not significant \\
\hline & 32 & Observer & FOS-IIIR noncompliance - targeted & Not significant \\
\hline & & Observer & FOS-IIIR noncompliance - general & Significant benefit \\
\hline & & Observer & FOS-IIIR Oppositional Behavior - targeted & Significant benefit \\
\hline & & Observer & FOS-IIIR Oppositional Behavior - general & Not significant \\
\hline & & Observer & FOS-IIIR Appropriate Behavior - targeted & Not significant \\
\hline & & Observer & FOS-IIIR Appropriate Behavior - General & Not significant \\
\hline \multirow{3}{*}{$\begin{array}{l}\text { Sanders et al. } \\
\text { [65] }\end{array}$} & 255 & Father & $\mathrm{ECBI}$ & Significant benefit \\
\hline & & Father & Parent Daily Report & Significant benefit \\
\hline & & Observer & $\begin{array}{l}\text { Revised Family Observation Schedule (FOS-RIII). } \\
\text { Composite score for negative child behavior }\end{array}$ & Not significant \\
\hline Stallman \& & 36 & Teenagers & Conflict behavior questionnaire & Not significant \\
\hline
\end{tabular}


Table 3 Child based outcomes reported by informants other than the child?'?s mother. (Continued)

\begin{tabular}{|c|c|c|c|c|}
\hline $\begin{array}{l}\text { Turner \& } \\
\text { Sanders [68] }\end{array}$ & 25 & $\begin{array}{l}\text { Independent } \\
\text { observers }\end{array}$ & Family Observation Schedule (disruptive behaviors) & Not significant \\
\hline \multirow{3}{*}{$\begin{array}{l}\text { Turner et al. } \\
\text { [29] }\end{array}$} & 21 & Father & Child behavior checklist & Not significant \\
\hline & & Observer & Mealtime observation & Not significant \\
\hline & & Observer & Anthropometric measures & Not significant \\
\hline
\end{tabular}

${ }^{a}$ Method of analysis not clearly specified. Reported as two-sample t-tests comparing intervention and control counties, presumably of the differences between pre- and post-randomization outcome variables. However, a stratified randomization was used, so the within-pair differences in the change from baseline should be analyzed, though these are not reported. CBCL, Child Behavior Checklist; ECBI, Eyberg Child Behavior Inventory; SDQ, Strengths and Difficulties Questionnaire.

of development of non-pharmaceutical interventions but readers should interpret findings accordingly, particularly when authors may gain financially from the intervention under study. Although authors of Triple P interventions receive royalty payments from sales of training and materials [27], only one of the articles we obtained declared any conflict of interest. Conflict of interest may be of particular importance in interpreting studies (such as many of those reported here) in which subgroup analyses are reported [40]. Outcome reporting bias [41] may also be an important consideration in this respect, with possible significance for the interpretation of meta-analyses [42].

Claims that whole-population parenting programs have significant impact on public health are particularly important, because these may have led to substantial commitment of public funds. We were unable to find any convincing evidence of benefit from the Triple $P$ program in the three whole-population studies eligible for inclusion in the present review.

\section{Summary of evidence}

Although a standard meta-analysis confirmed previous findings that mothers report improved child behavior after Triple-P interventions in comparison to a waiting list control condition, fathers and independent observers generally do not report improvements that are significantly different from those attributable to the control condition (Table 4). There is an absence of evidence of sustained benefit from Triple P interventions compared to control conditions, and no evidence that Triple $\mathrm{P}$ is superior to any other active intervention.

\section{Limitations}

Given the highly specific nature of the literature search, and the multiple sources of data, we believe that we have retrieved almost all of the relevant literature. We did not synthesize papers in languages other than English. We were not able to retrieve some book chapters with titles indicating possible eligible studies, but we did not find any new data reported in the many chapters we were able to retrieve. We did not obtain potentially relevant data from studies which were conducted but not subsequently published [30,36].

\section{Conclusions}

The studies to date give proof-of-concept that groupbased Triple-P may be effective in the short term according to maternal report of child behavior but, given the high risk of bias (or unknown risk of bias when reporting is poor), they do not support the view that Triple P provides other benefits to children.

The lack of convincing evidence of benefit from wholepopulation interventions is in line with previous work in which no significant improvement in child-based outcomes resulted from a public health parenting program [43] and with a more recent large-scale independent

Table 4 Papers giving paternally-reported ECBI Intensity scores.

\begin{tabular}{|c|c|c|c|c|c|c|c|}
\hline Author & Significantbenefit $(P<0.05) ?$ & $\mathrm{n} 1$ & mean1 & sd1 & $\mathrm{n} 2$ & mean2 & sd2 \\
\hline Bodenmann et al. [32] $]^{a}$ & No & 50 & 109.2 & 18.5 & 50 & 110.1 & 25.2 \\
\hline Connell et al. [50] & Yes & 11 & 154.55 & 17.44 & 12 & 111.0 & 12.41 \\
\hline Hahlweg et al. [51]* & No & 16 & 10.7 & 7.0 & 18 & 7.7 & 5.1 \\
\hline Hahlweg et al. [13]* & No & 57 & 9.3 & 7.2 & 141 & 10.2 & 6.9 \\
\hline Markie-Dadds \& Sanders [56] & No & \multicolumn{6}{|c|}{ NOT REPORTED } \\
\hline Morawska \& Sanders [61] & No & 24 & 111.57 & 20.41 & 49 & 106.07 & 24.37 \\
\hline Sanders et al. [65] & Yes & 71 & 127.34 & 22.39 & 184 & 113.13 & 27.34 \\
\hline
\end{tabular}

${ }^{\mathrm{a} E C B I}$ subscale data reported in [32] were assumed to have been transposed, and are corrected here. In this paper attrition rates at the post-treatment assessment are unknown and we assumed they remained constant. $\mathrm{n} 1$, mean 1 and sd1 are, respectively, group size, mean and standard deviation for the control groups, and $\mathrm{n} 2$, mean 2 and sd2 are the corresponding figures for the Triple-P intervention groups. Means and standard deviations are for ECBI-I subscale data, apart from the two papers marked with an asterisk, where the CBCL-E was reported. No paternally-reported data are tabulated in Markie-Dadds and Sanders [47], but there is a statement that 'Analyses of father-reported measures of child behavior failed to produce any significant effects.' CBCL-E, Child Behavior Checklist Externalizing scale; ECBI-I, Eyberg Child Behavior Inventory - Intensity scale. 
evaluation of Triple P in Zurich which demonstrated no impact on child behavior [44]. Along with findings from a previous systematic review [12], the results of our meta-regression support the view that some benefit might be achieved if interventions were focussed on the families of children with more severe problems. A recent Cochrane review of parent training interventions for children with established conduct problems and those at high risk of conduct disorder [45] provides robust evidence of the effectiveness of such targeted programs. It is, therefore, likely that an effective case-finding approach combined with offers of interventions to families with identified problems may be more effective than the 'public-health' approach.

Only one of the studies [26] included in our review had no apparent conflict of interest. We are aware of two further independent evaluations of Triple $\mathrm{P}$ which were ineligible for inclusion in our review - one published in German [46] and one recent large scale trial [44]. Both produced negative results. These findings mirror the frequently observed failure of independent replication of positive results from a range of developer-led studies. Theoretical models developed by Eisner [47], describing the mechanisms by which conflict of interest can lead to research bias, may help to explain this phenomenon.

Given the substantial cost implications, health care providers and policymakers would be well advised to apply the same standard of evidence when purchasing behavioral interventions as they do to the purchase of pharmaceutical agents or medical devices. Compulsory clinical trial registration and full and open declaration of conflicts of interest would address many of the deficits noted in our review. Pending the implementation of such mechanisms, unproven interventions should only be carried out in the context of a robust independent evaluation.

Developers and evaluators of psychological interventions should be encouraged to adhere to the guidelines dealing with good publication practice for communicating company sponsored medical research (GPP2 [48]). Journal editors and reviewers should be encouraged to adhere to CONSORT guidelines for both text and abstracts [49]: we believe that authors who choose journals that do not adhere to these guidelines, and editors who choose not to adopt them, do the field, as well as their own work, a disservice. There should be a clear expectation that instrument subscales should be reported in full and covered even-handedly in article abstracts. Care providers and policy makers should assess the generalizability of findings from socially advantaged and volunteer samples to their own situation. There is a need for registered, large, multicenter trials, with prospectively defined, long-term outcomes and active comparison groups rather than further evaluations using waiting list control groups. Rigorous systematic reviews of parenting interventions (for example [45]) attest to the importance of including data from independent observers in trials and in reviews, both in order to reduce risk of bias and to provide more convincing data on the effects of parenting interventions. Whole-population data on child behavior, reported by multiple informants, and linked to provision of parenting interventions, may be an alternative approach to the evaluation of public health parenting programs.

\section{Funding}

The study was unfunded, apart from a contribution made by the Gillberg Neuropsychiatry Centre to pay for statistical analysis. The funder played no part in the study design, analysis or interpretation of the data except insofar as one of the authors (CG) is employed within the Gillberg Neuropsychiatry Centre at the Sahlgrenska Academy, University of Gothenburg.

\section{Additional material}

Additional file 1: Main characteristics of the included studies.
Tabulation, for all eligible studies [5,6,13,20-29,32,33,50,51,53-69], of
numbers and characteristics of patients or families included, whether
problems were likely to be in the clinical range, inter-group comparisons
made, nature of the control group, length of follow-up and nature of
informants.

\section{Abbreviations}

CBCL: Child Behavior Checklist; CONSORT: Consolidated Standards of Reporting Trials; ECBI-I: Eyberg Child Behavior Inventory - Intensity scale; ES: effect size; PRISMA: Preferred Reporting Items for Systematic Reviews and Meta-Analysis; RCT: randomized controlled trial; SMD: standardized mean difference; Triple P: Positive Parenting Program.

\section{Acknowledgements}

We wish to thank Irene O'Neill for administrative support. Professor Manuel Eisner kindly provided information on conflicts of interest.

\section{Author details}

${ }^{1}$ Centre for Rural Health, University of Aberdeen, Centre for Health Sciences, Old Perth Rd, Inverness IV2 3JH, Scotland. Department of Health Sciences, Queen Margaret University, Queen Margaret University Drive, Musselburgh EH21 6UU, Scotland. ${ }^{3}$ Cromarty Medical Practice, Allan Square, Cromarty, Ross-shire IV11 8YF, Scotland. ${ }^{4}$ Institute of Health and Wellbeing, University of Glasgow, Caledonia House, Royal Hospital for Sick Children, Dalnair St, Yorkhill, Glasgow G3 8SJ, Scotland. ${ }^{5}$ Robertson Centre for Biostatistics, University of Glasgow, Boyd Orr Building, University Avenue, Glasgow G12 $8 \mathrm{QQ}$, Scotland.

\section{Authors' contributions}

PW, CP, PD, FS, SH and CG reviewed the published papers, RR performed the statistical analyses and AMCC provided statistical support to the authors. PW is guarantor of this paper. All authors contributed to the drafting of the article and approved the final submission.

\section{Competing interests}

PW is academic advisor to the evaluation of the Glasgow Parenting Support Framework, which has Triple P as one of its core components. AMcC provides statistical advice to this evaluation. Along with AMcC and CP, PW is a co-investigator in a NIHR funded trial comparing an antenatal Triple $P$ intervention with another parenting program, Mellow Parenting. CP is an 
author of the Mellow Parenting program, which is owned by a charity, and she is employed as a trainer for this program. Other authors report no conflicts of interest.

Received: 14 June 2012 Accepted: 2 November 2012 Published: 2 November 2012

\section{References}

1. Barlow J, Parsons J, Stewart-Brown S: Systematic review of the effectiveness of parenting programmes in the primary and secondary prevention of mental health problems Health Services Research Unit, University of Oxford; 2002.

2. Sanders MR, Markie-Dadds C, Turner K: Theoretical, Scientific and Clinical Foundations of the Triple-P - Positive Parenting Program: A Population Approach to the Promotion of Parenting Competence Brisbane: Australian Academic Press; 2003, 1-21, Parenting Research and Practice Monographs.

3. Sanders MR, Murphy-Brennan M: Creating conditions for success beyond the professional training environment. Clin Psychol: Sci Pract 2010, 17:31-35.

4. Sanders MR, Kirby JN: Parental programs for preventing behavioral and emotional problems in children. In Oxford Guide to Low Intensity CBT Interventions. Edited by: Bennet-Levy J, Richards D, Farrand P, Christensen H, Griffiths K, Kavanagh D, Klein B, Lau M, Proudfoot J, Ritterband L, White J, Williams C. New York, NY. Oxford University Press; 2010:399-406.

5. Prinz RJ, Sanders MR, Shapiro CJ, Whitaker DJ, Lutzker JR: Population-based prevention of child maltreatment: the U.S. Triple $\mathrm{p}$ system population trial. Prev Sci 2009, 10:1-12.

6. Sanders MR, Ralph A, Sofronoff K, Gardiner P, Thompson R, Dwyer S, Bidwell K: Every family: a population approach to reducing behavioral and emotional problems in children making the transition to school. J Prim Prev 2008, 29:197-222.

7. Dean C, Myors K, Evans E: Community-wide implementation of a parenting program: The South East Sydney Positive Parenting Project. AeJAMH (Australian e-Journal for the Advancement of Mental Health) 2003, 2:2003.

8. NICE. Conduct disorder in children - parent-training/education programmes. National Institute for Clinical Excellence; 2006 [http://www. nice.org.uk/TA102]

9. Nowak C, Heinrichs N: A comprehensive meta-analysis of Triple P-Positive Parenting Program using hierarchical linear modeling: effectiveness and moderating variables. Clin Child Fam Psychol Rev 2008, 11:114-144.

10. de Graaf I, Speetjens P, Smit F, de Wolff M, Tavecchio L: Effectiveness of the Triple P Positive Parenting Program on parenting: a meta-analysis. Fam Relat 2008, 57:553-566.

11. Thomas R, Zimmer-Gembeck MJ: Behavioral outcomes of parent-child interaction therapy and Triple P-Positive Parenting Program: a review and meta-analysis. J Abnorm Child Psychol 2007, 35:475-495.

12. de Graaf I, Speetjens P, Smit F, de Wolff M, Tavecchio L: Effectiveness of the Triple P Positive Parenting Program on behavioral problems in children: a meta-analysis. Behav Modif 2008, 32:714-735.

13. Hahlweg K, Heinrichs N, Kuschel A, Bertram H, Naumann S: Long-term outcome of a randomized controlled universal prevention trial through a positive parenting program: is it worth the effort? Child Adolesc Psychiatry Ment Health 2010, 4:14.

14. Liberati A, Altman DG, Tetzlaff J, Mulrow C, Götzsche PC, loannidis JPA, Clarke M, Devereaux P J, Kleijnen J, Moher D: The PRISMA statement for reporting systematic reviews and meta-analyses of studies that evaluate healthcare interventions: explanation and elaboration. BMJ 2009, 339: b2700, doi: 10.1136/bmj.b2700.

15. Parenting and Family Support Centre Triple-P database. University of Queensland;[http://www.pfsc.uq.edu.au/publications/].

16. de Graaf I, Speetjens P, Smit F, de Wolff M, Tavecchio L: Effectiveness of the Triple P Positive Parenting Program on parenting: A meta-analysis. Fam Relat 2008, 57:553-566.

17. Eyberg S, Ross AW: Assessment of child behaviour problems: the validation of a new inventory. J Clin Child Psychol 1978, 7:113-116.

18. Achenbach T, Edelbrock C: Manual for the Child Behavior Checklist and Revised Child Behavior Profile Burlington: University of Vermont; 1983.

19. Scottish Intercollegiate Guideline Network checklists. [http://www.sign.ac. $\mathrm{uk} /$ methodology/checklists.html].

20. Zubrick SR, Ward KA, Silburn SR, Lawrence D, Williams AA, Blair E, Robertson D, Sanders MR: Prevention of child behavior problems through universal implementation of a group behavioral family intervention. Prev Sci 2005, 6:287-304.

21. McTaggart P, Sanders MR: The Transition to School Project: Results from the classroom. Australian e-Journal for the Advancement of Mental Health 2003, 2:1-12.

22. West F, Sanders MR, Cleghorn GJ, Davies PSW: Randomised clinical trial of a family-based lifestyle intervention for childhood obesity involving parents as the exclusive agents of change. Behav Res Ther 2010, 48:1170-1179.

23. Wiggins TL, Sofronoff K, Sanders MR: Pathways triple P-positive parenting program: effects on parent-child relationships and child behavior problems. Fam Process 2009, 48:17-530.

24. Whittingham K, Sofronoff K, Sheffield J, Sanders MR: Stepping Stones Triple $P$ : an RCT of a parenting program with parents of a child diagnosed with an Autism Spectrum Disorder. J Abnorm Child Psychol 2009, 37:469-480.

25. Stallman HM, Ralph A: Reducing risk factors for adolescent behavioural and emotional problems: a pilot randomised controlled trial of a selfadministered parenting intervention. Australian e-Journal for the Advancement of Mental Health 2007, 6:1-13.

26. Gallart SC, Matthey S: The effectiveness of Group Triple P and the impact of the four telephone contacts. Behav Change 2005, 22:71-80.

27. Sanders MR, Stallman HM, McHale M: Workplace Triple P: a controlled evaluation of a parenting intervention for working parents. J Fam Psychol 2011, 25:581-590.

28. Nicholson JM, Sanders MR: Randomized controlled trial of behavioral family intervention for the treatment of child behavior problems in stepfamilies. J Divorce Remarriage 1999, 30:1-23.

29. Turner KMT, Sanders MR, Wall CR: Behavioural parent training versus dietary education in the treatment of children with persistent feeding difficulties. Behav Change 1994, 11:242-258.

30. Prinz RJ, Sanders MR: Adopting a population-level approach to parenting and family support interventions. Clin Psychol Rev 2007, 27:739-749.

31. Campbell MK, Elbourne DR, Altman DG: CONSORT statement: extension to cluster randomised trials. BMJ 2004, 328:702-708.

32. Bodenmann G, Cina A, Ledermann T, Sanders MR: The efficacy of the Triple P-Positive Parenting Program in improving parenting and child behavior: a comparison with two other treatment conditions. Behav Res Ther 2008, 46:411-427.

33. Roberts C, Mazzucchelli T, Studman L, Sanders MR: Behavioral family intervention for children with developmental disabilities and behavioral problems. J Clin Child Adolesc Psychol 2006, 35:180-193.

34. Turner KMT, Sanders MR, Wall CR: Behavioural parent training versus dietary education in the treatment of children with persistent feeding difficulties. Behav Change 1994, 11:242-258.

35. Foster $\mathrm{N}$, Little $\mathrm{P}$ : Methodological issues in pragmatic trials of complex interventions in primary care. Br J Gen Pract 2012, 62:10-11.

36. Ross MK, de Caestecker L: The Starting Well Health Demonstration Project: the best possible start in life? Int J Ment Health Promot 2009, 5:5-12.

37. Hutchings J, Gardner F, Bywater T, Daley D, Whitaker C, Jones K, Eames C, Edwards RT: Parenting intervention in Sure Start services for children at risk of developing conduct disorder: pragmatic randomised controlled trial. BMJ 2007, 334:678-685.

38. Gardner F, Burton J, Klimes I: Randomised controlled trial of a parenting intervention in the voluntary sector for reducing child conduct problems: outcomes and mechanisms of change. J Child Psychol Psychiatry 2006, 47:1123-1132.

39. Whittingham K, Sofronoff K, Sheffield J, Sanders MR: Do parental attributions affect treatment outcome in a parenting program? An exploration of the effects of parental attributions in an RCT of Stepping Stones Triple P for the ASD population. Res Autism Spectr Disord 2009, 3:129-144.

40. Sun X, Briel M, Busse JW, You JJ, Akl EA, Mejza F, Bala MM, Bassler D, Mertz D, Diaz-Granados N, Vandvik PO, Malaga G, Srinathan SK, Dahm P, Johnston BC, Alonso-Coello P, Hassouneh B, Truong J, Dattani ND, Walter SD, Heels-Ansdell D, Bhatnagar N, Altman D G, Guyatt GH: The influence of study characteristics on reporting of subgroup analyses in randomised controlled trials: systematic review. BMJ 2011, 342:d1569, doi: 10.1136/bmj.d1569.

41. Boutron I, Dutton S, Ravaud P, Altman DG: Reporting and interpretation of randomized controlled trials with statistically nonsignificant results for primary outcomes. JAMA 2010, 303:2058-2064. 
42. Roseman M, Milette K, Bero LA, Coyne JC, Lexchin J, Turner EH, Thombs BD: Reporting of conflicts of interest in meta-analyses of trials of pharmacological treatments. JAMA 2011, 305:1008-1017.

43. Hiscock H, Bayer JK, Price A, Ukoumunne OC, Rogers S, Wake M: Universal parenting programme to prevent early childhood behavioural problems: cluster randomised trial. BMJ 2008, 336:318-321.

44. Malti T, Ribeaud D, Eisner M: The effectiveness of two universal preventive interventions in reducing children's externalizing behavior: a cluster randomized controlled trial. J Clin Child Adolesc Psychol 2011, 40:677-692.

45. Furlong M, McGilloway S, Bywater T, Hutchings J, Smith S, Donnelly M: Behavioural and cognitive-behavioural group-based parenting programmes for early-onset conduct problems in children aged 3 to 12 years. Issue 2. Art. No.: CD008225. DOI: 10.1002/14651858.CD008225. pub2. Cochrane Database Syst Rev 2012, 2:CD008225.

46. Eisner M, Ribeaud D, Jünger R, Meidert U: Frühprävention von Gewalt und Aggression; Ergebnisse des Zürcher Interventions- und Präventionsprojektes an Schulen. Zürich: Rüegger; 2007.

47. Eisner M: No effects in independent prevention trials: can we reject the cynical view? J Exp Criminol 2009, 1:163-183.

48. Graf C, Battisti WP, Bridges D, Bruce-Winkler V, Conaty JM, Ellison JM, Field EA, Gurr JA, Marx ME, Patel M, Sanes-Miller C, Yarker YE: Good publication practice for communicating company sponsored medical research: the GPP2 guidelines. BMJ 2009, 339:b4330, doi: 10.1136/bmj. b4330.

49. Hopewell S, Ravaud P, Baron G, Boutron I: Effect of editors' implementation of CONSORT guidelines on the reporting of abstracts in high impact medical journals: interrupted time series analysis. BMJ 2012, 344:e4178, doi: 10.1136/bmj.e4178.

50. Connell S, Sanders MR, Markie-Dadds C: Self-directed behavioral family intervention for parents of oppositional children in rural and remote areas. Behav Modif 1997, 21:379-408.

51. Hahlweg K, Heinrichs N, Kuschel A, Feldmann M: Therapist-assisted, selfadministered bibliotherapy to enhance parental competence: short- and long-term effects. Behav Modif 2008, 32:659-681.

52. Kuschel A, Heinrichs $N$, Hahlweg $K$ : Is a preventive parenting program effective in reducing a child's externalizing behavior? Eur J Dev Sci 2009, 3:299-303.

53. Hoath FE, Sanders M: A feasibility study of enhanced group Triple P Positive Parenting Program for parents of children with attention-deficit/ hyperactivity disorder. Behav Change 2002, 19:191-206.

54. Joachim S, Sanders MR, Turner KMT: Reducing preschoolers' disruptive behaviour in public with a brief parent discussion group. Child Psychiatry Hum Dev 2010, 41:47-60.

55. Leung C, Sanders MR, Leung S, Mak R, Lau J: An outcome evaluation of the implementation of the Triple P-Positive Parenting Program in Hong Kong. Fam Proc 2003, 42:531-544.

56. Markie-Dadds C, Sanders M: A controlled evaluation of an enhanced selfdirected behavioural family intervention for parents of children with conduct problems in rural and remote areas. Behav Change 2006, 23:55-72.

57. Markie-Dadds C, Sanders M: Self-directed Triple P (Positive Parenting Program) for mothers with children at-risk of developing conduct problems. Behav Cogn Psychother 2006, 34:259-275.

58. Martin AJ, Sanders MR: Balancing work and family: a controlled evaluation of the Triple P-Positive Parenting Program as a work-site intervention. Child Adolesc Ment Health 2003, 8:161-169.

59. Matsumoto $Y$, Sofronoff K, Sanders M: The efficacy and acceptability of the Triple P-Positive Parenting Program with Japanese parents. Behav Change 2007, 24:205-218.

60. Matsumoto Y, Sofronoff K, Sanders MR: Investigation of the effectiveness and social validity of the Triple P Positive Parenting Program in Japanese society. J Fam Psychol 2010, 24:87-91.

61. Morawska A, Sanders MR: Self-administered behavioral family intervention for parents of toddlers: Part I. Efficacy. J Consult Clin Psychol 2006, 74:10-19.

62. Morawska A, Sanders M: An evaluation of a behavioural parenting intervention for parents of gifted children. Behav Res Ther 2009, 47:463-470.
63. Morawska A, Haslam D, Milne D, Sanders MR: Evaluation of a brief parenting discussion group for parents of young children. J Dev Behav Pediatr 2011, 2:136-145.

64. Plant KM, Sanders MR: Reducing problem behavior during care-giving in families of preschool-aged children with developmental disabilities. Res Dev Disabil 2007, 28:362-385.

65. Sanders MR, Markie-Dadds C, Tully LA, Bor W: The triple P-positive parenting program: a comparison of enhanced, standard, and selfdirected behavioral family intervention for parents of children with early onset conduct problems. J Consult Clin Psychol 2000, 68:624-640.

66. Sanders MR, Montgomery DT, Brechman-Toussaint ML: The mass media and the prevention of child behavior problems: the evaluation of a television series to promote positive outcomes for parents and their children. J Child Psychol Psychiatry 2000, 41:939-948.

67. Turner KM, Richards M, Sanders MR: Randomised clinical trial of a group parent education programme for Australian indigenous families. J Paediatr Child Health 2007, 43:429-437.

68. Turner KM, Sanders MR: Help when it's needed first: a controlled evaluation of brief, preventive behavioral family intervention in a primary care setting. Behav Ther 2006, 37:131-142.

69. Bor W, Sanders MR, Markie-Dadds C: The effects of the Triple P-Positive Parenting Program on preschool children with co-occurring disruptive behavior and attentional/hyperactive difficulties. J Abnorm Child Psychol 2002, 30:571-587.

\section{Pre-publication history}

The pre-publication history for this paper can be accessed here: http://www.biomedcentral.com/1741-7015/10/130/prepub

doi:10.1186/1741-7015-10-130

Cite this article as: Wilson et al:: How evidence-based is an 'evidencebased parenting program'? A PRISMA systematic review and metaanalysis of Triple P. BMC Medicine 2012 10:130.

\section{Submit your next manuscript to BioMed Central and take full advantage of:}

- Convenient online submission

- Thorough peer review

- No space constraints or color figure charges

- Immediate publication on acceptance

- Inclusion in PubMed, CAS, Scopus and Google Scholar

- Research which is freely available for redistribution 